\title{
Plasmonic Enhancement of Graphene Long-Wave Infrared Photodetectors via Bull's Eye Concentrator, Optical Cavity and Nanoantennas
}

\author{
Nima Sefidmooye Azar ${ }^{1}$, Vivek Raj Shrestha ${ }^{1}$, Kenneth B. Crozier ${ }^{1,2}$ \\ 1. Department of Electrical and Electronic Engineering, University of Melbourne, Victoria 3010, Australia \\ 2. School of Physics, University of Melbourne, Victoria 3010, Australia
}

The long-wave infrared photodetector device we study in this work is shown in Fig. 1 (a) and (b). A bull's eye grating collects the incident light from a large area $(101 \mu \mathrm{m})$, and couples it into surface plasmon polaritons (SPPs) [1] that converge at its center, at which there is an opening in the Au plate on which the grating sits. Within this opening (or aperture), Au nanobar antennas separated by small gaps are arranged in a radial fashion. These enhance the electromagnetic field in the near-field zone, particularly in the gaps, through localized surface plasmon resonances (LSPRs) [2]. This enhances the absorption in the underlying material, here a small circle (6.32 $\mu \mathrm{m}$ diam.) of graphene. The absorption is boosted further by forming an optical cavity under the graphene. We optimized the geometrical parameters to maximise absorption in the graphene at $\lambda=9.4 \mu \mathrm{m}$ via finitedifference time-domain electromagnetic simulations. These geometric parameters are listed in Fig. 1 (c)-(f).
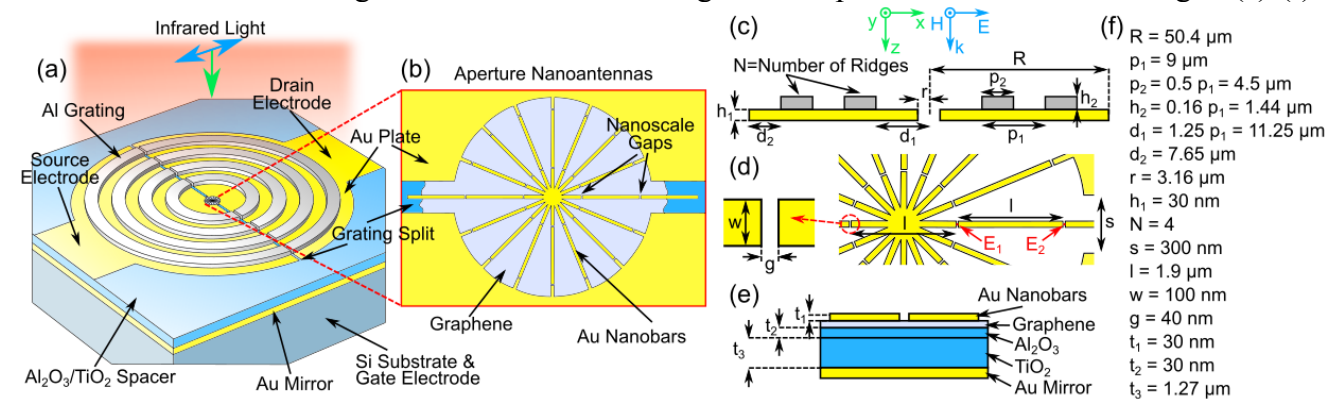

Fig. 1 (a) Cartoon illustrating the proposed LWIR photodetector consisting of a hybrid plasmonic structure. (b) Zoomed view of the aperture nanobar antennas. (c)-(f) Geometrical parameters of the structure.

Fig. 2 (a) presents the electric field enhancement at points $E_{1}$ and $E_{2}$, whose locations are defined in Fig. 1 (d). We observe an electric field enhancement of $\sim 380$ and $\sim 150$ times at $E_{1}$ and $E_{2}$, respectively, at the resonance wavelength $(\lambda=9.4 \mu \mathrm{m})$. Fig. 2 (b) plots normalized power absorbed in graphene vs wavelength, which peaks at $\sim 1.63 \%$ at the resonance wavelength. In this figure, the normalized power absorbed is the power absorbed in the graphene divided by the power incident through a square window with a side length of $101 \mu \mathrm{m}$.
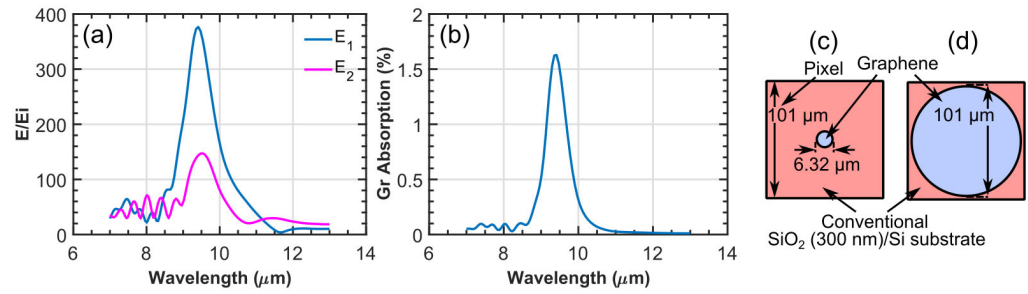

Fig. 2 (a) Electric field enhancement at points $E_{1}$ and $E_{2}$, respectively. $E_{i}$ is the norm of the incident electric field. (b) Normalized power absorbed in graphene as a function of wavelength. (c) and (d) Schematic of the non-plasmonic device on a conventional $\mathrm{SiO}_{2} / \mathrm{Si}$ substrate with a small-area and large-area graphene flake, respectively.

Our structure is predicted to enhance the absorption of light by the graphene by $\sim 4600$ times compared with its non-plasmonic counterpart (Fig. 2 (c)). It is predicted to enhance detectivity of the LWIR photodetector by $\sim 288$ times compared with its non-plasmonic graphene counterpart (Fig. 2 (d)). This estimate is made using $D^{*}=$ $\sqrt{A_{O} \Delta f} / N E P$ [3]. This assumes that the photoactive material is coupled to the incident light by an optical concentrator with area $A_{O}$, with $\triangle f$ and $N E P$ being bandwidth and noise equivalent power. NEP is assumed to scale as $\sqrt{A_{D}} /\left(\right.$ Gr abs), where $A_{D}$ is graphene area and Gr abs is normalized power absorbed in the graphene.

\section{References}

[1] E. Wijaya, C. Lenaerts, S. Maricot, J. Hastanin, S. Habraken, J. P. Vilcot, R. Boukherroub, and S. Szunerits, "Surface Plasmon Resonance-Based Biosensors: From the Development of Different SPR Structures to Novel Surface Functionalization Strategies," Curr. Opin. Solid State Mater. Sci. 15, 208-224 (2011).

[2] V. Giannini, A. I. Fernandez-Dominguez, S. C. Heck, and S. A. Maier, "Plasmonic Nanoantennas: Fundamentals and Their Use in Controlling the Radiative Properties of Nanoemitters," Chem. Rev. 111, 3888-3912 (2011).

[3] J. Piotrowski, "Uncooled Operation of IR Detectors," Opto-Electron. Rev. 12, 111-122 (2004). 


\section{University Library}

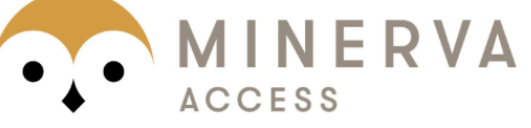

A gateway to Melbourne's research publications

Minerva Access is the Institutional Repository of The University of Melbourne

Author/s:

Azar, NS;Shrestha, VR;Crozier, KB

Title:

Plasmonic Enhancement of Graphene Long-Wave Infrared Photodetectors via Bull's Eye Concentrator, Optical Cavity and Nanoantennas

Date:

2019-01-01

\section{Citation:}

Azar, N. S., Shrestha, V. R. \& Crozier, K. B. (2019). Plasmonic Enhancement of Graphene Long-Wave Infrared Photodetectors via Bull's Eye Concentrator, Optical Cavity and Nanoantennas. [Abstract]. 2019 Conference on Lasers and Electro-Optics Europe / European Quantum Electronics Conference (CLEO/Europe-EQEC), Part F143-EQEC 2019, https://doi.org/10.1109/CLEOE-EQEC.2019.8872291.

Persistent Link:

http://hdl.handle.net/11343/294877 